\title{
BINARY STUCTURE OF ACCRETING NEUTRON STARS
}

\author{
D.A. LEAHY \\ University of Calgary \\ Calgary, Alberta, Canada T2N 1N4
}

\section{Introduction}

The study of X-ray binaries has made great progress with the advent in the past few years of a number of very capable X-ray astronomy missions. These are reviewed, for example, by Bradt et al 1992, and a set of recent relevant papers in Makino and Mitsuda, 1997. For example, ASCA has allowed a significant increase in sensitivity and spectral resolution in $0.5-10 \mathrm{keV}$ $\mathrm{X}$-rays (Tanaka et al 1994). Many recent Compton/GRO results on X-ray binaries are reviewed in the proceedings of the Second Compton Symposium (Fichtel et al 1994). Another source of recent results from analysis of data from several satellite missions is the proceedings of the Evolution of X-ray Binaries (Holt \& Day, 1994). In this short paper, the emphasis is on guiding the reader to some relevant literature.

\section{Theory and Observations of Accreting Neutron Stars}

The source of energy is gravitational potential energy. Various thermalization processes occur which result in temperatures for the X-ray emitting region of several keV (e.g. Joss \& Rappaport 1984, Parmar, 1994). Material can flow slowly from the companion through the Lagrangian point to form a large disk around the compact object. Or, if the companion is well within the Roche lobe, a stellar wind can cause material to flow at high speed past the Lagrangian point and form a small disk. The small disk can be transient and reverse its spin in a quasiperiodic manner (Fryxell \& Taam, 1988, Blondin et al, 1991). The disk interacts with the magnetosphere of the neutron star, then the infalling matter is thermalized in an accretion mound at the surface or by a strong shock above the neutron star surface. The opacity is dominated by the cyclotron opacity (e.g. Meszaros and Nagel, 1985). 
Prince et al (1994) gives a recent review of BATSE results on X-ray pulsars, in particular his Table 2 lists orbital parameters for $15 \mathrm{X}$-ray pulsars determined by pulse timing. The low mass systems show a regular spin down over the 3 years of monitoring. The intermediate mass system Her $\mathrm{X}-1$ shows a random walk behaviour in period. The high mass systems show a significant random walk component but also a secular trend: spin-up for Cen X-3 and spin-down for OAO 1657-415 and Vela X-1. The details of the spin behaviours are not understood, but are related to the accretion torque on the neutron star and the various components of angular momentum of the neutron star and their coupling forces.

Valuable results on the state of cicumstellar matter in Vela X-1 are given by Nagase 1994 (see also these proceedings for Vela X-1, GX301-2, Cen X-3 and other systems). New results also come from analysis of archival data. E.g., GINGA observations of Her X-1 give interesting results (Leahy, 1997, Scott, 1994).

In summary, numerous physical processes can be studied, ranging from the dynamics of neutron stars to conditions in circumstellar and accreting plasmas (temperatures, ionization states, elemental abundances). Current and future observations of this nature are giving further insight into the structure and physical state of accretion flows in X-ray binaries.

\section{References}

Blondin, J., Kallman, T., Fryxell, B. \& Taam, R. 1990, ApJ, 356, 591

Bradt, H., Ohashi, T. \& Pounds, K. 1992, ARAA, 30, 391

Fichtel, C., Gehrels, N. \& Norris, J. 1994, Proceedings of the Second Compton Symposium (AIP Conference Proceedings 304)

Fryxell, B. \& Taam, R. 1988, ApJ, 335, 862

Holt, S. \& Day, C. 1994, Proceedings of the Evolution of X-ray Binaries (AIP Conference Proceedings 306)

Joss, P. \& Rappaport, S. 1984, ARAA, 22, 537

Leahy, D. 1997, M.N.R.A.S. 287, 622

"X-ray Imaging and Spectroscopy of Cosmic Hot Plasmas" ed. Makino, F., Mitsuda, K. (Universal Academy Press, Tokyo, 1997)

Meszaros, P. \& Nagel, W. 1985, ApJ, 299, 138

Nagase, F. 1994, in Proceedings of the Evolution of X-ray Binaries (AIP Conference Proceedings 306), p567

Parmar, A. 1994 in Proceedings of the Evolution of X-ray Binaries (AIP Conference Proceedings 306), p415

Prince, T. et al 1994 in Proceedings of the Evolution of X-ray Binaries (AIP Conference Proceedings 306), p235

Scott, J.M. 1994 PhD Thesis (Washington State University)

Tanaka, Y., Inoue, H. \& Holt, S. 1994, PASJ, 46, L37 